\title{
FEASIBILITY OF THE NONLINEAR BEAM-BEAM COMPENSATION AT TEVATRON
}

\author{
Y.Alexahin, V.Shiltsev, FNAL, Batavia, IL 60510, \\ D.Shatilov, BINP, Novosibirsk, Russia
}

\section{Abstract}

It is planned to overcome the major limitation imposed by the beam-beam effect on the achievable luminosity in Tevatron with the help of electron lenses [1]; the primary goal being to eliminate the p-bar bunch-to-bunch tunespread due to the PACMAN effect (linear beam-beam compensation). The possibility of the simultaneous compression - by adjusting the electron beam profile - of the intrabunch nonlinear tunespread (nonlinear compensation) is considered. Major limitations on the achievable degree of compression are analyzed.

\section{INTRODUCTION}

Owing to a number of reasons - a larger number of protons per bunch and a smaller proton emittance, a factor of seven larger number of the parasitic long-range interactions - the beam-beam driven resonances (the synchro-betatron ones in particular) can present in the Tevatron Run II configuration [2] a greater danger for the stability of antiprotons than previously [3].

Another complication associated with the parasitic interactions is the so-called PACMAN effect: dependence of the tuneshifts on the bunch position in the train. As shown in Ref. [4] the bunch-to-bunch tunespread can be completely eliminated with the help of two linear lenses created by electron beams of constant charge density (linear beam-beam compensation). To ensure that all antiprotons see a linear force from the electron beam its radius taken in the antiproton beam r.m.s. radius should be sufficiently large (at least $3 \sigma$ ), still this does not guarantee the stability of tail particles which loss may lead to a high level of background.

There are several disadvantages inherent to the linear beam-beam compensation (BBC):

- the intrabunch nonlinear tunespread is much larger than the bunch-to-bunch tunespread being compensated; therefore it is still difficult to accommodate the total

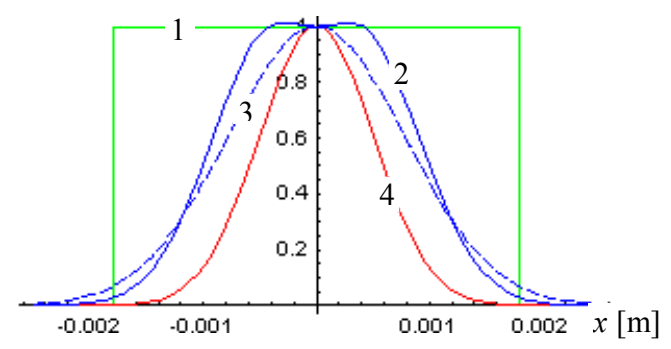

Figure 1: Space charge distribution: 1 - linear TEL, 2 - TEL with "profiler", 3 - Gaussian distribution with the same current, 4 - antiproton beam at TEL1. footprint in a resonance free area;

- there is strong excitation of high order resonances for antiprotons with large betatron amplitudes which see the steep edges of the electron beam;

- the electron beam current, owing to a large beam radius, should be high $(\geq 6 \mathrm{~A})$ easily reaching the electrostatic limit in the Tevatron beam pipe [1]; a highcurrent electron beam may also produce an unwanted effect on the protons.

These problems can be simultaneously solved by shaping the electron beam in the gun with a Pierce-like electrode [5] (a "profiler") so that it has a smaller r.m.s. size and smoother edges as shown in Fig. 1 with the solid blue line.

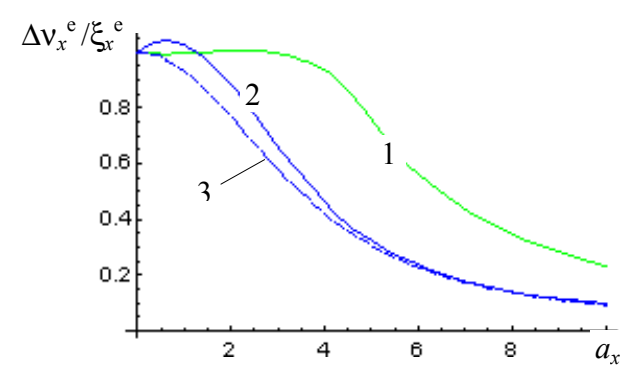

Figure 2: Normalized horizontal tuneshift experienced by antiprotons due to TEL with e-beam profiles as shown in Fig. 1 vs. betatron amplitude in units of the antiproton beam r.m.s. radius.

\section{NONLINEAR TUNESHIFT}

With the electron and antiproton beam sizes becoming comparable, there appears a strong amplitude dependence of the tuneshifts produced by a Tevatron electron lens (TEL) as shown in Fig.2. It counteracts the tuneshift with amplitude resulting from the head-on collisions with the proton beam thus leading to a compression of the total tunespread within a single antiproton bunch (the nonlinear beam-beam compensation).

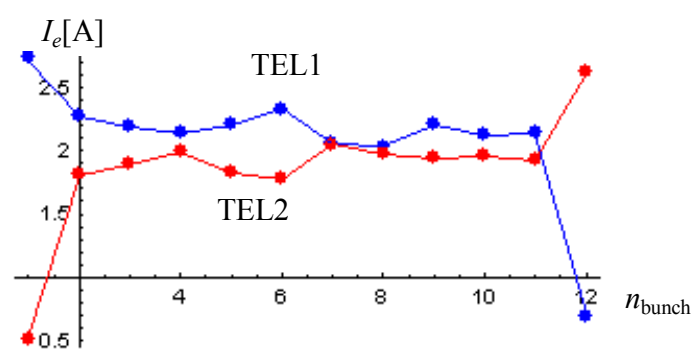

Figure 3: Electron currents in the two TELs as seen by different antiproton bunches. 


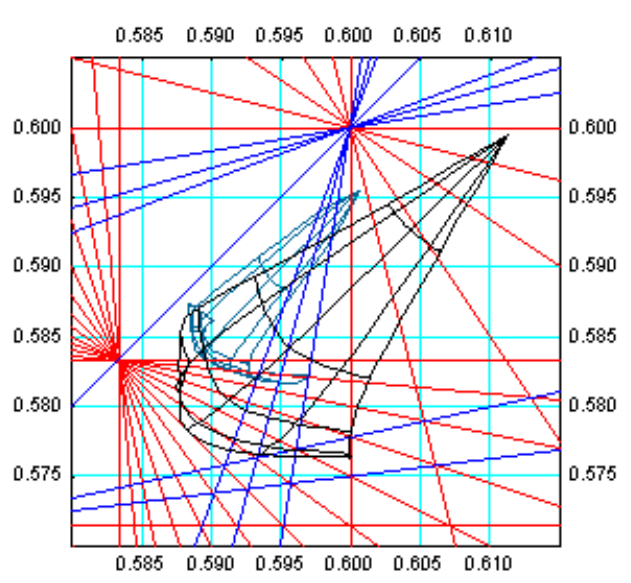

Figure 4: Original and compressed antiproton bunch \#6 footprint in the vicinity of the nominal working point.

Obviously, to benefit from such compensation one should eliminate the bunch-to-bunch tunespread first, so two electron lenses are necessary: TEL1 at location with larger horizontal $\beta$-function $\left(\beta_{x}=98.7 \mathrm{~m}, \beta_{y}=28.4 \mathrm{~m}\right)$ and TEL2 at location with larger vertical $\beta$-function $\left(\beta_{x}=56.7 \mathrm{~m}, \beta_{y}=172 \mathrm{~m}\right)$.

To begin with we have chosen the electron beam sizes (HWHM) to be $r_{e}=1 \mathrm{~mm}$ in TEL1 and $r_{e}=1.3 \mathrm{~mm}$ in TEL2. The electron currents which provide a complete compensation of the bunch-to-bunch tunespread and compress the intra-bunch nonlinear tunespread by a factor of two are shown in Fig.3. Due to smaller e-beam sizes they are twice lower than needed for the linear BBC [4].

The effect of TELs on the nonlinear tunespread is illustrated by Fig. 4, which shows the antiproton bunch \#6 footprint in the tune diagram without BBC (black) and with it (teal blue). The bare lattice tunes (assumed nominally to be $v_{x}=20.585, v_{y}=20.575$ ) were slightly trimmed in the latter case. The arc lines correspond to equidistant with step 2 values of the total transverse amplitude

$$
a_{\perp}=\sqrt{a_{x}^{2}+a_{y}^{2}},
$$

where $a_{x, y}$ are taken in the pbar $\sigma$ 's, the radial lines correspond to constant values of $a_{x} / a_{y}$. The red and blue lines show respectfully sum and difference resonances of orders lower than 13.

It can be seen that the footprint "folding", which is caused by the long-range interactions with the proton beam and without $\mathrm{BBC}$ happens at amplitudes $\sim 8 \sigma$, with $\mathrm{BBC}$ takes place at amplitudes as low as $5 \sigma$. Since even very weak high order resonances may lead to a fast particle transport over the region of folding thus reducing the pbars lifetime, this effect sets a natural limit on the degree of the footprint compression.

Another limitation comes from the requirement that the tune modulation by the synchrotron motion due to finite dispersion at the TEL locations was small: $r_{e}>>D_{x} \sigma_{E} \approx 1.7 \mathrm{~m} \times 9 \cdot 10^{-5} \approx 0.15 \mathrm{~mm}$, to avoid strong excitation of the synchrotron satellites of betatron resonances.

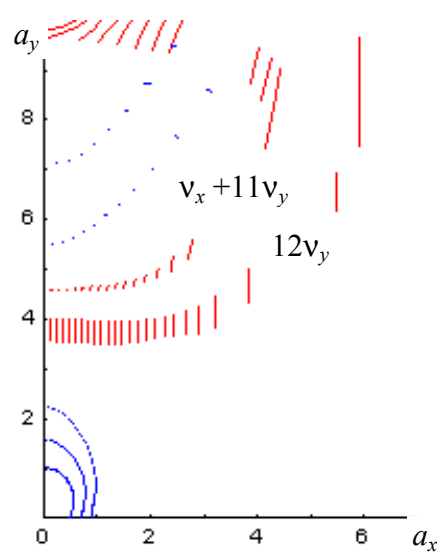

Figure 5: Swing of the betatron amplitudes due to resonances encountered by pbars of bunch $\# 6$ at the nominal working point with $\mathrm{BBC}$.

\section{RESONANCE EXCITATION}

The electron beams of TELs can themselves contribute to excitation of resonances. Fig. 5 shows beatings of the betatron amplitudes (calculated analytically in a single resonance approximation) due to resonances encountered by antiprotons of bunch \#6 whose footprint with BBC was shown in Fig. 4 in teal blue.

Due to the TEL contribution the width of the $12 v_{y}$ resonance is much larger than that of the $v_{x}+11 v_{y}$ resonance which, in the absence of misalignments, is excited exclusively by the long-range interactions.

For off-momentum particles the effective resonance width is even larger owing to the synchrotron satellites.

\subsection{Choice of the working point}

The compressed with TELs footprint can fit into other areas in the tune diagram which are surrounded by less dangerous resonances. One such possibility, with the tunes around $v_{x}=20.563, v_{y}=20.557$, was considered for the linear BBC [6]. However, this area is not wide enough to avoid setting some particles on either $7^{\text {th }}$ or $9^{\text {th }}$ order resonances. Another option is the SPS working point. The compressed with TELs footprint at the bare lattice tunes $v_{x}=20.689, v_{y}=20.682$ (which we refer to as the SPS WP) is shown in Fig.6.

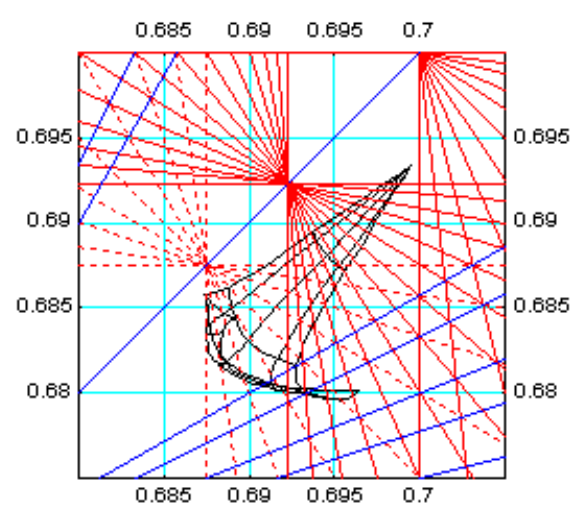

Figure 6: Bunch \#6 footprint at the SPS WP with BBC by two Gaussian TELs 


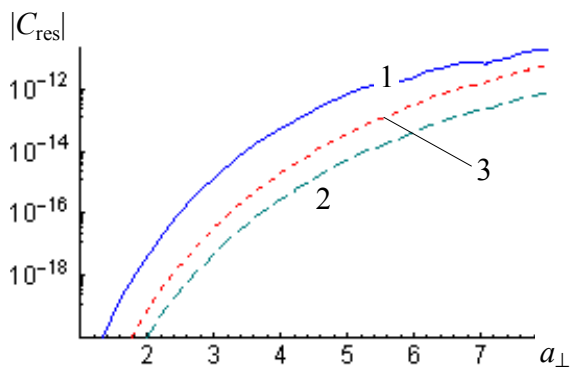

Figure 7: The $10 v_{x}+6 v_{y}$ resonance driving term for $a_{x}=$ $a_{y}=a_{\perp} / 2^{1 / 2}: 1-2$ TELs with flat-top e-beam (Fig.1, curve 2), 2 - 2 TELs with Gaussian e-beam, 3 - 1 TEL with Gaussian e-beam at location with $\beta_{\mathrm{r}}=\beta_{v}$.

\subsection{Effect of the electron beam profile}

Excitation of the $16^{\text {th }}$ order resonances (and its satellites) by TELs can facilitate diffusion of antiprotons, especially in the region of amplitudes where the footprint folding occurs. As the example of $10 v_{x}+6 v_{y}$ resonance shows (Fig.7), excitation of high order resonances by TELs can be reduced by making the e-beam charge distribution more monotonous (e.g. Gaussian). One more advantage is that the footprint folding occurs at somewhat larger amplitudes with the Gaussian e-beams (compare the compressed footprints in Figs.4 and 6).

\subsection{Effect of the number of TELs}

There is an additional argument (besides compensation of the PACMAN effect) in favor of using two TELs at points with $\beta_{x}>>\beta_{y}$ and $\beta_{x}<<\beta_{y}$ rather than one TEL at a location with equal $\beta$ 's. From the resonances excited by TELs the high-order sum resonances are the most dangerous (the WP can always be chosen so that the unidimensional ones were reached at too small amplitudes to be noticeable or not reached at all). Since the driving term of the $k v_{x}+l v_{y}=n$ resonance contains the factor $\beta_{x}^{k / 2} \beta_{y}^{l / 2}$, its excitation is significantly suppressed in the case of two TELs, as illustrated by Fig.7 (curves 2,3). In all cases TELs provided the same horizontal tuneshift $\xi_{x}{ }^{e}=-0.014$.

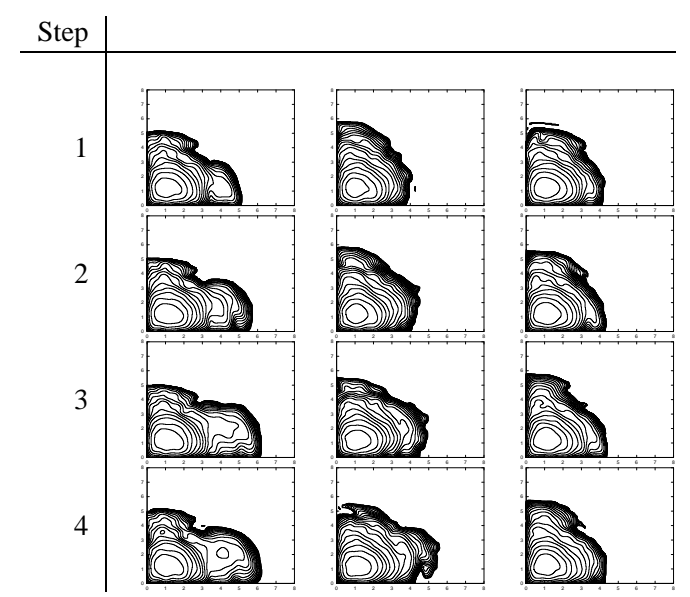

Figure 8: Evolution (from top to bottom) of the density of antiprotons in the $\left(a_{x}, a_{y}\right)$ space with step $10^{5}$ turns in the cases described in the text.

\subsection{Effect of the finite dispersion}

Owing to the finite dispersion TELs can contribute to excitation of the synchro-betatron resonances (SBRs) two-fold: via the tune modulation arising from the steep fall-off of the instantaneous tuneshift with the displacement $D_{x} \delta_{p}$, and via variation of the nonlinear component of the TEL field seen by an antiproton in the course of the synchrotron motion.

According to the estimates the second effect should not be important, whereas the first one, the TEL second order chromaticity, is big: TEL tuneshift is modulated by more than $20 \%$ at $a_{s}=3$. Both effects can be reduced, if necessary, by increasing the e-beam radius.

\section{TRACKING SIMULATIONS}

All the above presented results were obtained in the single resonance approximation using analytical formulae of Ref.[3]. More realistic picture can be obtained by tracking simulations with the code LIFETRAC which can take into account the cooperative action all beam-beam and TEL nonlinearities and the external noise.

Fig. 8 shows evolution of the bunch \#6 density at the SPS WP with the BBC by two Gaussian TELs which provide zero-amplitude tuneshifts of $\Delta v_{x}{ }^{e} \approx-0.0144$, $\Delta v_{y}{ }^{\mathrm{e}} \approx-0.0115$. With the bare lattice tunes $20.689,20.682$ the $13^{\text {th }}$ order resonances proved to be strong enough to affect the core particles (left column). With tunes shifted down by 0.005 (center column) the core was not affected, still some tails had developed which were not seen in the test run with linear lenses instead of TELs. Weakening the TELs nonlinearity by a $15 \%$ increase in the e-beam sizes diminishes the tails (right column). In all three cases neither luminosity nor lifetime had suffered.

\section{CONCLUSIONS}

- The degree of nonlinear BBC is limited by the footprint "folding" due to the long-range contribution and the electron lens chromaticity due to finite dispersion.

- The possibility to eliminate the footprint folding by additional compensation of the long-range interactions (e.g. with pulsed wires) should be studied.

- Excitation of high order resonances by TELs can be reduced by choosing a smooth electron beam charge distribution (e.g. Gaussian) and using two TELs at points with strongly unbalanced $\beta$-functions.

- The compression of the footprint by a factor of two permits to fit it in the areas free of resonances of orders lower than 13, the neighborhood of the SPS working point being a promising candidate.

\section{REFERENCES}

[1] V.Shiltsev et al., this conference.

[2] http://www-bd.fnal.gov/runII/index.html

[3] Y.Alexahin, FERMILAB-TM-2148 (2001)

[4] V.Shiltsev et al., PRST-AB, v.2, 071001 (1999)

[5] A.Shemyakin et al., Proc. EPAC 2000, Vienna, p.1271.

[6] Y.Alexahin et al., this conference. 